\title{
EDUCACIÓN
}

\section{Fundamentos para la aplicación de estándares internacionales en la formación del Licenciado en Educación de la Lengua Inglesa en la Educación Superior de Gestión Pública del Paraguay. 2017}

\author{
Dora Alicia Velázquez Moreno
}

\begin{abstract}
Resumen
Introducción: La ausencia de estándares internacionales, en la formación del licenciado en Educación de Lengua Inglesa en la institución de ES de gestión pública plantea una realidad disociante en cuanto a los planes nacionales de desarrollo vigentes. La certificación de niveles de dominio de la lengua Inglesa, europeas o norteamericanas, en la formación de licenciados establece un nuevo paradigma de calidad ante las exigencias de internalización de la educación superior, la cooperación de la ciencia, la cultura, el mercado laboral y la industria del país y con respecto a la región misma; su ausencia desfavorece la competitividad de los egresados con respecto a sus pares nacionales e internacionales.
\end{abstract}

Objetivo: Diagnosticar las condiciones actuales del Plan de Estudios de la licenciatura en Educación de la Lengua Inglesa en la Educación Superior de gestión pública (Instituto Superior de Educación).

Material y Método: Investigación descriptiva inicial, de naturaleza empírica y documental, sustentada en fuentes primarias y secundarias, de diseño transversal, enfoque cualicuantitativo. La observación directa, apoyada en las técnicas de análisis de contenido del Plan de estudios de la carrera, el estándar europeo Marco Común de Referencia para las lenguas (MCER), la aplicación de encuestas y entrevista enfocada a la población de docentes del turno mañana/ tarde, referente académica de la carrera, estudiantes del primer y último año, turnos mañana/tarde.

Resultados: En el Plan de Estudios de la licenciatura, y/o caracterización de la carrera no existe tipificación de niveles de dominio de la lengua inglesa en los perfiles de ingreso, egreso o campo ocupacional/profesional que determine un nivel de dominio de las competencias comunicativas de la lengua inglesa de

1. UNIBE. Facultad de Humanidades. Ciencias de la Educación.

Tesis para la obtención del grado de Magister del Programa en Planificación y Conducción Estratégica Nacional en el Instituto de Altos Estudios Estratégicos-Consejo de Defensa Nacional Presidencia de la República-Asunción, Noviembre 2017.

E-mail: ways.py@hotmail.com

DOI: $10.26885 /$ rcei.foro. 2018.50 
calidad, sean éstas oral, auditiva, lectora y escrita, en relación al estándar europeo MCER comparado ni otro parámetro internacional especificado o sugerido. Tampoco se registra o sugiere el grado de adecuación de nivel de dominio de la Lengua Inglesa en el enfoque metodológico de enseñanza- aprendizaje para el desarrollo de las competencias: lingüística, sociolingüística y pragmática mínimas; las estrategias docentes empleadas quedan individualizadas según la decisión y/o percepción de los docentes.

Conclusión: La ausencia de estándares internacionales en el Plan de estudios, con respecto a otros de países del MERCOSUR, a nivel regional, y continental consultados y descriptos en el Marco Referencial establecen convenientemente en los mismos, perfiles de ingreso y egreso, lo que deriva a una exigencia de nivel de dominio de calidad a docentes no nativos de la lengua. La tendencia actual hacia la economía de conocimientos en Educación Superior, y en particular aquellas de gestión pública, determinan un nuevo modelo de gestión, activando mecanismos de rendición de cuentas para justificar el gasto público. Las limitaciones derivadas del déficit de estándares internacionales en el plan de estudios de esta carrera son amplias, desmeritando la inversión social en detrimento del desarrollo nacional.

Palabras clave: educación superior, gestión pública, pertinencia, estándar europeo MCER, gasto público.

\section{Referencias}

Cárdenas, M. (2009). Tendencias globales y locales en la formación de docentes de lenguas extranjeras. IKALA Revista de lenguaje y Cultura, 14(22),71-103. Recuperado de http://www.bdigital.unal.edu. co/40014/1/79638758.2014.pdf

COUNCIL OF EUROPE. (2011). Common European Framework of reference for languages: learning, teaching, assessment. Cambridge University Press. Recuperado de https://www.coe.int/t/dg4/linguistic/source/ framework_en.pdf

Instituto Superior de Educación. (sf). Plan de la carrera de Licenciatura en educación de la Lengua Inglesa. Recuperado de http://www. ise.edu.py/index.php/carrera/carreras/carreras-de-grado/270/ licenciatura-en-educacion-de-la-lengua-inglesa

PARAGUAY. CONES. (2017). Libro blanco de la educación superior. Recuperado de http://www.cones.gov.py/ libro-blanco-para-la-educacionsuperior/

PARAGUAY. Plan de Desarrollo Nacional 2030. Recuperado de http://www.stp. gov.py/pnd/wpcontent/uploads/2014/12/pnd2030.pdf 